QUARTERLY OF APPLIED MATHEMATICS

VOLUME LXVIII, NUMBER 4

DECEMBER 2010, PAGES 747-763

S $0033-569 X(2010) 01202-2$

Article electronically published on October 15, 2010

\title{
PIEZO-VISCOUS FLOWS OVER AN INCLINED SURFACE
}

\author{
BY \\ G. SACCOMANDI (Dipartimento di Ingegneria Industriale, Università di Perugia, Via G. Duranti, \\ 06125 Italy) \\ AND
}

L. VERGORI (Dipartimento di Matematica, Università del Salento, Strada Prov. Lecce-Arnesano, 73100 Lecce, Italy)

\begin{abstract}
Stokes was the first to recognize that the viscosity of many fluids varies significantly with pressure. Later, several experimental studies showed that such a variation may be exponential. Here, by using the lubrication theory as revised by Rajagopal and Szeri, we study the flow of a piezo-viscous fluid down an incline in various flow regimes.
\end{abstract}

1. Introduction. At normal operating conditions, the viscosity of an incompressible liquid is assumed to be independent of the pressure. However, it is well known that the viscosity of a fluid can change with pressure, and if the pressure range is significantly large the viscosity can change by several orders of magnitude. In his celebrated paper on the response of fluids, Stokes [23] notes that the viscosity of a fluid could depend upon the pressure. However, based on the experiments of Du Buat on the flow of water in canals and pipes under normal operating conditions, Stokes suggested that the viscosity could be considered to be a constant for such flows. Stokes is however very careful to delineate the class of flows wherein the viscosity might be considered to be a constant and he also remarks that such an assumption would be invalid under other flow conditions. In many organic liquids, while the density might change by a few percent due to a significant change in the pressure, the viscosity, on the contrary, could change by many orders of magnitude (as much as a factor of $10^{8}$ !) (see, for instance, 15] and the references therein). Thus one could consider such liquids as incompressible fluids with pressure-dependent viscosities.

As early as 1893, Barus [4] proposed an empirical relationship between the viscosity and the pressure, namely

$$
\mu(p)=\mu_{0} \exp \left[\beta\left(p-p_{0}\right)\right],
$$

Received April 1, 2009.

2000 Mathematics Subject Classification. Primary 76A20; Secondary 76A02.

Key words and phrases. Lubrication approximation, pressure-dependent viscosity, Barus' law.

E-mail address: saccomandi@mec.dii.unipg.it

E-mail address: luigi.vergori@unile.it 
where $\mu_{0}$ is the viscosity at the pressure $p_{0}$ and $\beta$ is a piezoviscous coefficient that varies with temperature. Later, Andrade [2] suggested the following expression for the viscosity:

$$
\mu(p, \rho, \theta)=A \rho^{1 / 2} \exp \left[\left(p+\rho r^{2}\right) \frac{s}{T}\right],
$$

based on experiments. In the above expression, $\rho$ denotes the density, $T$ the temperature, $p$ the pressure, and $r, s$ and $A$ are constants. More recently, Laun [7] has modeled the viscosity of polymer melts through

$$
\mu(p, T)=\mu_{0} \exp \left[\beta\left(p-p_{0}\right)-\gamma\left(T-T_{0}\right)\right],
$$

where $\mu_{0}$ is the viscosity at pressure $p_{0}$ and temperature $T_{0}$, and $\beta$ and $\gamma$ are nonnegative constants. There have been numerous other experiments by Bair and co-workers that show that the dependence of the viscosity on the pressure is exponential (see recent experiments of Bair and Kottke [3]). Mention must be made of the work of MartínAlfonso and co-workers [10] wherein an intricate relationship between the temperature, viscosity and pressure is provided for bitumen. In this context, it ought to be pointed out that the pressure dependence of the properties of bitumen were recognized very early. For instance, Saal and Koens [21] not only allowed for viscosity to depend on pressure (the normal stress), they even allowed it to depend on the shear stresses. Thus, they had a truly implicit constitutive model relating the stress and kinematical quantities (see also [11] and [22]).

In recent years there has been a notable interest in piezo-viscous fluids. Rajagopal and co-workers $([6]-9],[14-20,24,, 26])$ have studied in detail several important and basic problems for such fluids. One example of a physical situation wherein the dependence of the viscosity on the pressure cannot be ignored is the problem of elastohydrodynamics [25]. In such a physical situation the dependence of the viscosity on the pressure is exponential so that the viscosity varies by several orders of magnitude for the range of the pressure that is relevant.

In this study, we investigate the gravity current resulting from the spread of a fluid with pressure-dependent viscosity down an incline of constant slope. To this end we use the well-established lubrication theory that is revised by Rajagopal and Szeri 20] to include the contributions to the equations of elastohydrodynamic lubrication due to variations in the viscosity with respect to the pressure. Since the main assumption in lubrication approximation is that the lengthscale ratio $\epsilon$ is small, i.e.,

$$
\epsilon=\frac{H}{L} \ll 1,
$$

and it is clear that if $H$ is too small there is no reason to consider the piezo-viscous model, we think that our study may be relevant to the geophysical applications of this theory such as gravity currents under water, ground water motions, lava flows (see for instance 12 and the references therein). Indeed, in such a framework it may be possible that the involved lengths $L$ are so important that for depths $H$ of significant thickness to appreciate pressure variations, $\epsilon$ is small enough.

Our results are complementary to those found by Rajagopal and Szeri in 20, where for the first time a consistent derivation of the equations of lubrication theory that takes into account the pressure dependence of viscosity has been derived. In [20] only steady state 
problems significant in important technical applications (such as the classical problem of an elastic cylinder rolling on a plane) are considered, whereas here we are interested in time-dependent problems with a free surface.

The plan of the paper is the following. In Section 2 we derive the basic equations and the corresponding boundary conditions. In Section 3 we study the nearly steady regime considering only gravity effects and gravity effects plus surface tension. Section 4 is devoted to the viscous regime. In particular, when surface tension effects can be neglected, we study the simple one-dimensional flow which is downslope or upslope. Besides the uniform solutions, various families of traveling wave solutions may be found by numerical integration. Concerning the gravity effects plus surface tension, we study the linear stability of the uniform solutions. The last section is devoted to concluding remarks.

2. Basic equations and boundary conditions. We consider a fluid moving on a nonhorizontal plane, whose angle of inclination is $\alpha$. Let $O x y z$ be a Cartesian frame of reference with fundamental unit vectors $\mathbf{i}, \mathbf{j}$ and $\mathbf{k}$, the coordinate $z$ being perpendicular to the plane, the $x$ and $y$ coordinates lying in the plane, $y$ being horizontal and $x$ increasing downward. We denote the components of the velocity of the fluid in the directions $x, y$ and $z$ as $u, v$ and $w$, respectively.

Let $h=h(x, y, t)$ be the free surface of the current, whose characteristic thickness and characteristic length along the plane we denote with $H$ and $L$, respectively. The main assumption in lubrication approximation is that the lengthscale ratio $\epsilon$ in (1.1) is small; therefore it is clear that the component of the velocity parallel to the plane is much larger than the normal component, so that

$$
\sqrt{u^{2}+v^{2}} \gg|w| .
$$

The equations of momentum balance and the equation of continuity can be written as

$$
\left\{\begin{array}{c}
\rho \dot{u}=-p_{x}+\mu(p)\left(u_{x x}+u_{y y}+u_{z z}\right) \\
+\mu^{\prime}(p)\left[2 u_{x} p_{x}+\left(u_{y}+v_{x}\right) p_{y}+\left(u_{z}+w_{x}\right) p_{z}\right]+\rho g \sin \alpha, \\
\rho \dot{v}=-p_{y}+\mu(p)\left(v_{x x}+v_{y y}+v_{z z}\right) \\
\quad+\mu^{\prime}(p)\left[\left(u_{y}+v_{x}\right) p_{x}+2 v_{y} p_{y}+\left(v_{z}+w_{y}\right) p_{z}\right], \\
\rho \dot{w}=-p_{z}+\mu(p)\left(w_{x x}+w_{y y}+w_{z z}\right) \\
\quad+\mu^{\prime}(p)\left[\left(u_{z}+w_{x}\right) p_{x}+\left(v_{z}+w_{y}\right) p_{y}+2 w_{z} p_{z}\right]-\rho g \cos \alpha \\
u_{x}+v_{y}+w_{z}=0
\end{array}\right.
$$

where $\rho$ is the density of the fluid, $p$ is the pressure, $\mu(p)$ is the pressure-dependent viscosity of the fluid, $g$ is the acceleration due to gravity and

$$
\mu^{\prime}(p)=\frac{\mathrm{d} \mu}{\mathrm{d} p}(p) .
$$

Here we shall mainly focus on Barus' law,

$$
\mu(p)=\mu_{0} \mathrm{e}^{\beta\left(p-p_{0}\right)}, \quad \mu_{0}>0, \beta>0,
$$


where $\mu_{0}$ is the fluid viscosity at the reference pressure $p_{0}$ and $\beta$ is the piezo-viscous coefficient. Other pressure-dependent fluid viscosity models that are largely studied in the literature (see for instance [17] and the references therein) are of the form

$$
\mu(p)=\mu_{0}+A\left(p-p_{0}\right)^{n}, \quad \mu_{0}>0, A>0, n>0,
$$

where, as before, $\mu_{0}$ is the fluid viscosity at the reference pressure $p_{0}$.

To system (2.1) we append the boundary conditions

$$
\begin{cases}u=v=0 & \text { at } z=0 \\ w=0 & \text { at } z=h, \\ u_{z}=v_{z}=0 & \text { at } z=h \\ w_{z z}=0 & \text { at } z=h \\ p=p_{0}+\gamma \operatorname{div}(\mathbf{n}) & \text { at } z=h\end{cases}
$$

where $\gamma$ is the surface tension and

$$
\mathbf{n}=\frac{1}{\sqrt{1+h_{x}^{2}+h_{y}^{2}}}\left(-h_{x} \mathbf{i}-h_{y} \mathbf{j}+\mathbf{k}\right)
$$

is the unit normal to the free surface of the current $z=h(x, y, t)$. The local curvature may thus be readily computed:

$$
\operatorname{div}(\mathbf{n})=-\frac{h_{x x}+h_{y y}+h_{x x} h_{y}^{2}+h_{y y} h_{x}^{2}-2 h_{x y} h_{x} h_{y}}{\left(1+h_{x}^{2}+h_{y}^{2}\right)^{3 / 2}}
$$

and hence the boundary condition (2.4) 4 becomes

$$
p=p_{0}-\gamma \frac{h_{x x}+h_{y y}+h_{x x} h_{y}^{2}+h_{y y} h_{x}^{2}-2 h_{x y} h_{x} h_{y}}{\left(1+h_{x}^{2}+h_{y}^{2}\right)^{3 / 2}} \text { at } z=h .
$$

There are many ways of transforming these governing equations and boundary conditions into dimensionless expressions. Here we introduce a scaling which is similar to that introduced by Ancey [1]:

$$
\left\{\begin{aligned}
\mathbf{x}^{*}=\frac{1}{L}(x \mathbf{i}+y \mathbf{j})+\frac{z}{H} \mathbf{k}, & \mathbf{v}^{*}=\frac{1}{U_{\|}}(u \mathbf{i}+v \mathbf{j})+\frac{w}{W} \mathbf{k} \\
W=\frac{H}{L} U_{\|}, \quad t^{*}=\frac{U_{\|}}{L} t, & p^{*}=\frac{p-p_{0}}{\rho g \cos \alpha H}, \quad \mu^{*}=\frac{\mu}{\mu_{0}}
\end{aligned}\right.
$$

where $\mu_{0}=\mu\left(p_{0}\right)$ is the viscosity of the fluid evaluated at the reference pressure $p_{0}$. Substituting the dimensionless quantities (2.6) into equations (2.1) and boundary conditions 
(2.4) and (2.5) leads to (omitting all asterisks)

$$
\left\{\begin{aligned}
\epsilon \operatorname{Re} \dot{u}=\frac{\epsilon \operatorname{Re}}{\operatorname{Fr}^{2}}\left(\frac{\tan \alpha}{\epsilon}-p_{x}\right)+\mu(p)\left[\epsilon^{2}\left(u_{x x}+u_{y y}\right)+u_{z z}\right] & \\
& +\mu^{\prime}(p)\left[\epsilon^{2}\left(2 u_{x} p_{x}+u_{y} p_{y}+v_{x} p_{y}+w_{x} p_{z}\right)+u_{z} p_{z}\right], \\
\epsilon \operatorname{Re} \dot{v}=-\frac{\epsilon \operatorname{Re}}{\operatorname{Fr}^{2}} p_{y}+\mu(p)\left[\epsilon^{2}\left(v_{x x}+v_{y y}\right)+v_{z z}\right] & \quad+\mu^{\prime}(p)\left[\epsilon^{2}\left(u_{y} p_{x}+v_{x} p_{x}+2 v_{y} p_{y}+w_{y} p_{z}\right)+v_{z} p_{z}\right], \\
\epsilon^{3} \operatorname{Re} \dot{w}=- & \frac{\epsilon \operatorname{Re}}{\operatorname{Fr}^{2}}\left(1+p_{z}\right)+\mu(p)\left[\epsilon^{4}\left(w_{x x}+w_{y y}\right)+\epsilon^{2} w_{z z}\right] \\
& +\mu^{\prime}(p)\left[\epsilon^{2}\left(u_{z} p_{x}+v_{z} p_{y}+2 w_{z} p_{z}\right)+\epsilon^{4}\left(w_{x} p_{x}+w_{y} p_{y}\right)\right], \\
u_{x}+v_{y}+ & w_{z}=0
\end{aligned}\right.
$$

with boundary conditions

$$
\begin{gathered}
u=v=0 \quad \text { at } z=0, \\
w=0 \quad \text { at } z=h, \\
u_{z}=v_{z}=0 \quad \text { at } z=h, \\
w_{z z}=0 \quad \text { at } z=h, \\
p=-\frac{\epsilon^{2}}{\mathrm{Bo}} \frac{h_{x x}+h_{y y}+\epsilon^{2}\left(h_{x x} h_{y}^{2}+h_{y y} h_{x}^{2}-2 h_{x y} h_{x} h_{y}\right)}{\left[1+\epsilon^{2}\left(h_{x}^{2}+h_{y}^{2}\right)\right]^{3 / 2}} \text { at } z=h,
\end{gathered}
$$

where

$$
\operatorname{Re}=\frac{\rho U_{\|} H}{\mu_{0}}, \quad \operatorname{Fr}=\frac{U_{\|}}{\sqrt{g \cos \alpha H}} \quad \text { and } \quad \mathrm{Bo}=\frac{\rho g \cos \alpha H^{2}}{\gamma}
$$

are the Reynolds, Froude and Bond numbers, respectively. The dimensionless version of the viscosity functions introduced in (2.2) and (2.3) takes the form

$$
\mu(p)=\mathrm{e}^{\lambda p} \quad \text { with } \quad \lambda=\beta \rho g \cos \alpha H
$$

and

$$
\mu(p)=1+\lambda p^{n} \quad \text { with } \quad \lambda=A(\rho g \cos \alpha H)^{n},
$$

respectively. Observe that the dimensionless viscosity tends to unity 1 as the dimensionless pressure tends to zero.

Depending on the values considered for the characteristic scales, different types of flow regime occur. In this paper we shall focus on the following two types of flow regime:

i) Nearly steady uniform regime, where the viscous contribution matches gravity acceleration. In this case, we have

$$
U_{\|}=\frac{\rho g \sin \alpha H^{2}}{\mu_{0}}
$$

and $\mathrm{Fr}^{2}=O(\mathrm{Re})$. Inertial terms and pressure gradient terms must be negligible, which means that $\epsilon \operatorname{Re} \ll 1$.

\footnotetext{
${ }^{1}$ In the classical case in which the fluid viscosity is a constant, the dimensionless viscosity is $\mu(p)=1$.
} 
ii) Viscous regime, where the pressure gradient is balanced by viscous stresses within the bulk. In this case, we have

$$
U_{\|}=\frac{\rho g \cos \alpha H^{3}}{\mu_{0} L}
$$

and consequently $\operatorname{Fr}^{2}=\epsilon \operatorname{Re}$. Inertial terms must be low compared to the pressure gradient and the slope must be gentle $(\tan \alpha=O(\epsilon))$. This imposes the following constraint: $\epsilon \operatorname{Re} \ll 1$.

Concerning the boundary condition for the pressure we observe that the surface tension effects can be neglected whenever Bo is of order $\epsilon$ or greater, whereas they must be retained if $\mathrm{Bo}=O\left(\epsilon^{2}\right)$. Therefore, in the former case, the boundary condition (2.12) approximates to

$$
p=0 \quad \text { at } z=h,
$$

whereas in the latter case, by virtue of assumption (1.1), (2.12) reduces to

$$
p=-\frac{\Delta h}{\overline{\mathrm{Bo}}} \quad \text { at } \quad z=h,
$$

where $\overline{\mathrm{Bo}}=\frac{\epsilon^{2}}{\mathrm{Bo}}$.

3. Nearly steady uniform regime. In this case the approximated governing equations are given by

$$
\left\{\begin{array}{l}
\frac{\partial\left[\mu(p) u_{z}\right]}{\partial z}+1=0, \\
\frac{\partial\left[\mu(p) v_{z}\right]}{\partial z}=0, \\
p_{z}+1=0, \\
u_{x}+v_{y}+w_{z}=0 .
\end{array}\right.
$$

In the following two subsections we shall study the gravity effects and the gravity effects plus surface tension on the nearly steady uniform flow regime.

3.1. Gravity effects only. It is easy to check that system (3.1) with boundary conditions (2.8)-(2.10) and (2.15) admits the solution

$$
\left\{\begin{array}{l}
u=\int_{0}^{z} \frac{h-\zeta}{\mu(h-\zeta)} \mathrm{d} \zeta \\
v=0 \\
p=h-z \\
w=h_{x}\left[\frac{h}{\mu(h)}(h-z)-\int_{z}^{h} \frac{h-\zeta}{\mu(h-\zeta)} \mathrm{d} \zeta\right] .
\end{array}\right.
$$

We define the average $u$ as

$$
\bar{u}=\frac{1}{h} \int_{0}^{h} u \mathrm{~d} z
$$




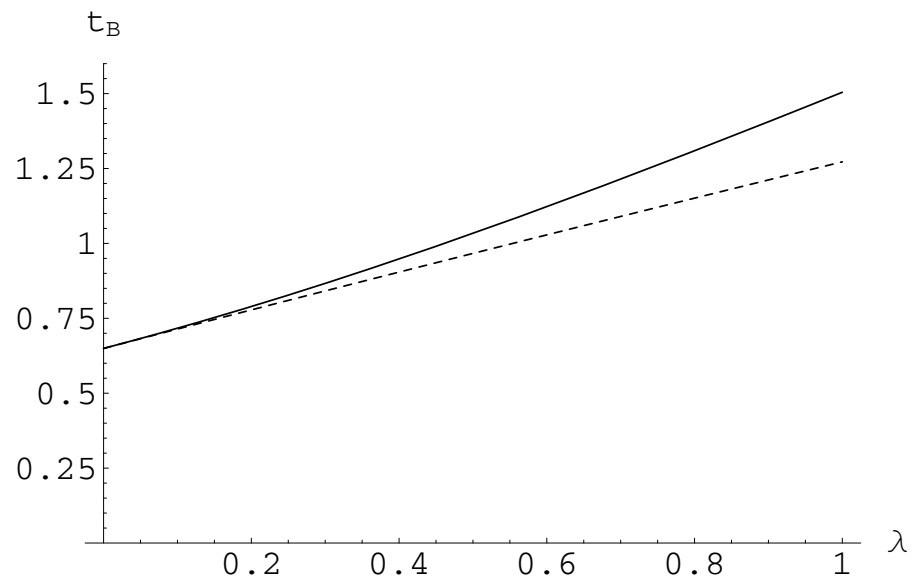

Fig. 1. Breaking time as a function of the nondimensional piezoviscous coefficient $\lambda$ when the dimensionless pressure-dependent viscosity is of the form (2.13) (solid line) and of the form (2.14) with $n=1$ (dashed line). The initial profile considered is $h(x, 0)=1-x^{2}$. In both cases, in the limit as $\lambda \rightarrow 0, t_{B}$ tends to $3 \sqrt{3} / 8$, that is, the breaking time in the classical case $\mu(p)=1$.

Then

$$
h \bar{u}=\int_{0}^{h} \frac{\xi^{2}}{\mu(\xi)} \mathrm{d} \xi .
$$

The mass conservation equation can be written in the form

$$
h_{t}+(h \bar{u})_{x}+(h \bar{v})_{y}=0 .
$$

Inserting (3.2) in equation (3.3) and noticing that $h \bar{v}=0$ yield that

$$
h_{t}+a(h) h_{x}=0,
$$

where

$$
a(h)=\frac{h^{2}}{\mu(h)} .
$$

Equation (3.4) is a quasilinear first-order partial differential equation whose general solution can be found by the method of characteristics. If $f(\xi)$ is an initial profile, then the corresponding solution is given by

$$
h=f(x-a(h) t) .
$$

The wave (3.5) could break at time $t_{B}=-1 / a^{\prime}\left(f\left(\xi_{B}\right)\right)$ at the point $x_{B}=\xi_{B}+a\left(f\left(\xi_{B}\right)\right) t_{B}$, where $\xi_{B}$ has to be determined by means of the conditions

$$
\left\{\begin{array}{l}
a^{\prime}\left(f\left(\xi_{B}\right)\right)<0, \\
\left|a^{\prime}\left(f\left(\xi_{B}\right)\right)\right|=\max \left|a^{\prime}(f(\xi))\right| .
\end{array}\right.
$$

In Figure 1 the breaking time is expressed as a function of the nondimensional piezoviscous coefficient $\lambda$ when the dimensionless viscosity is given by (2.13) and by (2.14) with $n=1$ under the initial condition $h(x, 0)=1-x^{2}$. 


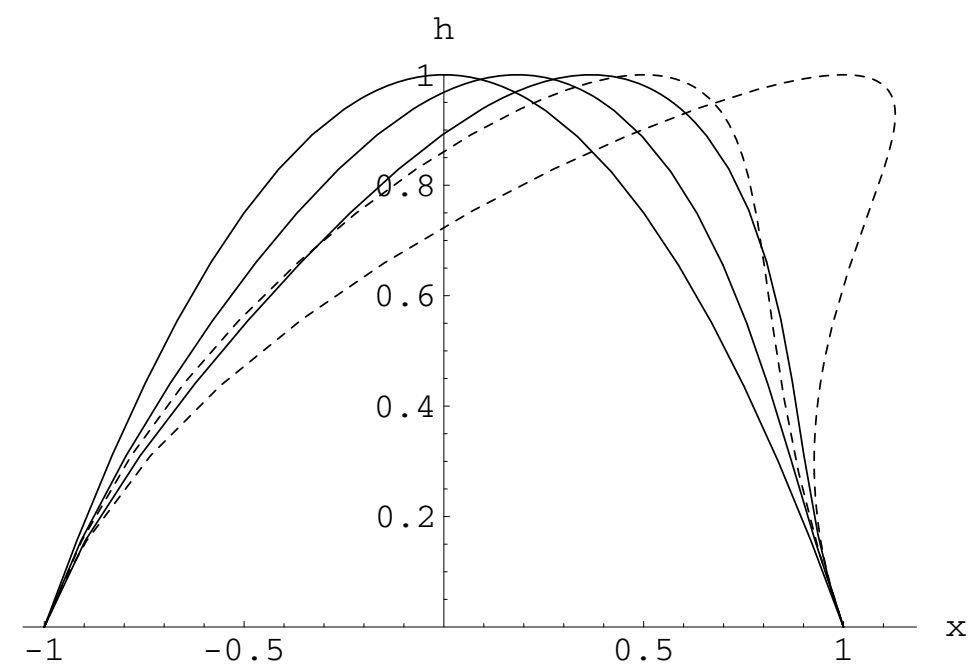

FIG. 2. Solutions of equation (3.4) with an initial profile $f(x)=$ $\left(1-x^{2}\right)$ for $t=0, t=0.5, t=1$. The dashed line represents the solution in the classical case $\mu(p)=1$, whereas the solid line represents the solution in the case in which the dimensionless fluid viscosity depends on the pressure according to (2.13) with $\lambda=1$.

3.2. Gravity and surface tension effects. If surface tension effects must be retained (if Bo $=O\left(\epsilon^{2}\right)$ ), then system (3.1) with boundary conditions (2.8)-(2.10) and (2.16) admits the solution

$$
\left\{\begin{array}{l}
u=\int_{0}^{z} \frac{h-\zeta}{\mu(h-\zeta-\Delta h / \overline{\mathrm{Bo}})} \mathrm{d} \zeta \\
v=0 \\
p=h-z-\frac{\Delta h}{\overline{\mathrm{Bo}}} \\
w=h_{x}\left[\frac{h}{\mu(h-\Delta h / \overline{\mathrm{Bo}})}(h-z)-\int_{z}^{h} \frac{h-\zeta}{\mu(h-\zeta-\Delta h / \overline{\mathrm{Bo}})} \mathrm{d} \zeta\right] .
\end{array}\right.
$$

Then

$$
h \bar{u}=\int_{0}^{h} \frac{\xi^{2}}{\mu(\xi-\Delta h / \overline{\mathrm{Bo}})} \mathrm{d} \xi,
$$

$h \bar{v}=0$ and the mass conservation equation (3.3) is given by

$$
h_{t}+\frac{\partial}{\partial x} \int_{0}^{h} \frac{\xi^{2}}{\mu(\xi-\Delta h / \overline{\mathrm{Bo}})} \mathrm{d} \xi=0 .
$$

We observe that when surface tension effects can be neglected, i.e. whenever Bo is of order $\epsilon$ or greater, $\mu=\mu(\xi)$ in the second term at the left-hand side of equation (3.6) so that equation (3.6) reduces to equation (3.4). We have already seen that the solution of (3.4) yields waves that travel in the direction of the shear and they steepen as they go. No instability is present. When surface tension is present we investigate the behaviour 
of small disturbances to the uniform solution $h=h_{0}$ of equation (3.6). We perturb the solution $h=h_{0}$ with a small disturbance $\tilde{h}$, periodic in $x: h=h_{0}+\tilde{h}$. Sustituting this into equation (3.6) yields

$$
\tilde{h}_{t}+\frac{h_{0}^{2}}{\mu\left(h_{0}\right)} \tilde{h}_{x}-\frac{1}{\overline{\mathrm{Bo}}}\left[\int_{0}^{h_{0}} \xi^{2} \frac{\mathrm{d}}{\mathrm{d} \xi}\left(\frac{1}{\mu(\xi)}\right) \mathrm{d} \xi\right] \tilde{h}_{x x x}=0 .
$$

Since this equation has coefficients independent of $t$ and $x$, we seek separable solutions of the form

$$
\tilde{h}=\tilde{h}_{0} \exp (\mathrm{i} k x+s t)
$$

which is a complete set of normal modes that can be used to represent any disturbance. By substituting these into equation (3.7) we obtain the following characteristic equation for $s$ :

$$
s=-\mathrm{i} k\left[\frac{h_{0}^{2}}{\mu\left(h_{0}\right)}+\frac{k^{2}}{\overline{\mathrm{Bo}}} \int_{0}^{h_{0}} \xi^{2} \frac{\mathrm{d}}{\mathrm{d} \xi}\left(\frac{1}{\mu(\xi)}\right) \mathrm{d} \xi\right] .
$$

Therefore any normal mode of the form (3.8) represents a wave which translates along the $x$-axis with nondimensional phase speed

$$
\operatorname{Im}\left(\frac{s}{k}\right)=-\left[\frac{h_{0}^{2}}{\mu\left(h_{0}\right)}+\frac{k^{2}}{\overline{\mathrm{Bo}}} \int_{0}^{h_{0}} \xi^{2} \frac{\mathrm{d}}{\mathrm{d} \xi}\left(\frac{1}{\mu(\xi)}\right) \mathrm{d} \xi\right] .
$$

From (3.9) we deduce that, in the classical case, the nondimensional phase speed is constant $\left(=-h_{0}^{2}\right)$ (see also [12]), whereas for fluids whose viscosity is pressure-dependent it depends quadratically on the dimensionless wave number $k$. Finally, each normal mode (3.8) is neutrally stable as $\operatorname{Re}(s)=0$ so that the amplitude of any small disturbance $\tilde{h}$ will remain small at any time.

4. Viscous regime. In this case, the approximated governing equations are given by

$$
\left\{\begin{array}{l}
\frac{\partial\left[\mu(p) u_{z}\right]}{\partial z}+\left(\frac{\tan \alpha}{\epsilon}-p_{x}\right)=0 \\
\frac{\partial\left[\mu(p) v_{z}\right]}{\partial z}-p_{y}=0 \\
p_{z}+1=0 \\
u_{x}+v_{y}+w_{z}=0
\end{array}\right.
$$

In the following two subsections we shall study the gravity effects and the gravity effects plus surface tension on the viscous flow regime. 
4.1. Gravity effects. It is easy to check that system (4.1) with boundary conditions (2.8)-(2.10) and (2.15) admits the solution

$$
\left\{\begin{array}{l}
u=\left(\frac{\tan \alpha}{\epsilon}-h_{x}\right) \int_{0}^{z} \frac{h-\zeta}{\mu(h-\zeta)} \mathrm{d} \zeta, \\
v=-h_{y} \int_{0}^{z} \frac{h-\zeta}{\mu(h-\zeta)} \mathrm{d} \zeta, \\
p=h-z \\
w=\int_{z}^{h} \nabla \cdot\left[\int_{0}^{\zeta_{1}} \frac{h-\zeta_{2}}{\mu\left(h-\zeta_{2}\right)} \mathrm{d} \zeta_{2}\left(\frac{\tan \alpha}{\epsilon} \mathbf{i}-\nabla h\right)\right] \mathrm{d} \zeta_{1} .
\end{array}\right.
$$

Then

$$
\begin{aligned}
& h \bar{u}=\left(\frac{\tan \alpha}{\epsilon}-h_{x}\right) \int_{0}^{h} \frac{\xi^{2}}{\mu(\xi)} \mathrm{d} \xi, \\
& h \bar{v}=-h_{y} \int_{0}^{h} \frac{\xi^{2}}{\mu(\xi)} \mathrm{d} \xi
\end{aligned}
$$

and consequently the mass conservation equation (3.3) is given by

$$
h_{t}+\nabla \cdot\left[\int_{0}^{h} \frac{\xi^{2}}{\mu(\xi)} \mathrm{d} \xi\left(\frac{\tan \alpha}{\epsilon} \mathbf{i}-\nabla h\right)\right]=0 .
$$

Now we assume that the flow depends only on the $x$ coordinate. Then $h_{y}=0$ (so that $v=0$ ) and equation (4.2) reduces to

$$
h_{t}+\frac{\partial}{\partial x}\left[\int_{0}^{h} \frac{\xi^{2}}{\mu(\xi)} \mathrm{d} \xi\left(\frac{\tan \alpha}{\epsilon}-h_{x}\right)\right]=0
$$

To find traveling wave solutions we assume that $h$ depends on the single variable $s \equiv$ $x-c t$, where $c$ is a constant which represents the wave speed. Then equation (4.3) can be integrated once to obtain

$$
\frac{\mathrm{d} h}{\mathrm{~d} s}=\frac{\tan \alpha}{\epsilon}-\frac{c_{1}+c h}{\int_{0}^{h} \frac{\xi^{2}}{\mu(\xi)} \mathrm{d} \xi},
$$

$c_{1}$ being an integration constant. We observe that

$$
\int_{0}^{h} \frac{\xi^{2}}{\mu(\xi)} \mathrm{d} \xi \simeq \frac{h^{3}}{3} \quad \text { as } \quad h \rightarrow 0
$$




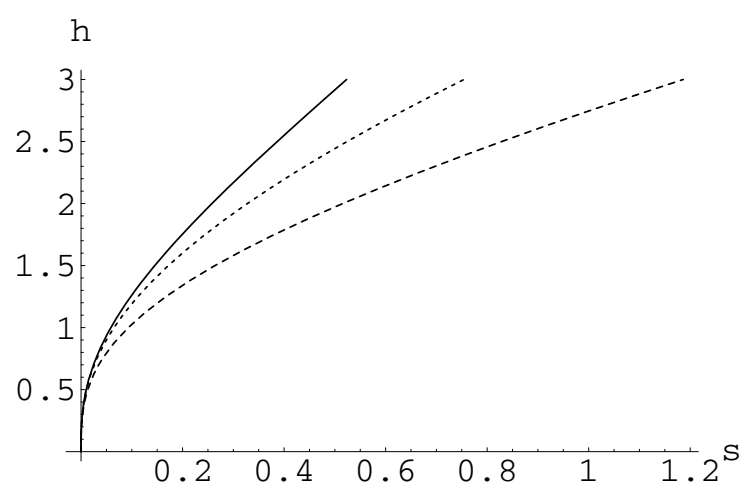

FIG. 3. Profiles of traveling waves moving upward in the classical case $\mu(p)=1$ (dashed line), in the presence of a linear dependence of viscosity upon pressure, $\mu(p)=1+p$ (dotted line), and in the presence of the nondimensional Barus' law $\mu(p)=\mathrm{e}^{p}$ (solid line). Here we have assumed that $c=-1, \tan \alpha=\epsilon$ and $c_{1}=c_{2}=0$.

Therefore at the wave front the slope of the free surface becomes infinite and, if $c_{1}=0$, as $h \rightarrow 0$ the solution of equation (4.4) can be approximated by

$$
s=\left\{\begin{array}{l}
\frac{\epsilon}{\tan \alpha}\left(h+\sqrt{\frac{3 c \epsilon}{\tan \alpha}} \ln \sqrt{\left.\left|\frac{h-\sqrt{\frac{3 c \epsilon}{\tan \alpha}}}{h+\sqrt{\frac{3 c \epsilon}{\tan \alpha}}}\right|\right)+c_{2} \quad \text { if } \quad c>0,}\right. \\
\frac{\epsilon}{\tan \alpha}\left[h-\sqrt{\frac{3|c| \epsilon}{\tan \alpha}} \arctan \left(\sqrt{\frac{\tan \alpha}{3|c| \epsilon}} h\right)\right]+c_{2} \quad \text { if } \quad c<0,
\end{array}\right.
$$

where $c_{2}$ is another integration constant. The approximated solution (4.5) is the solution of (4.4) with $c_{1}=0$ and dimensionless viscosity $\mu(p)=1$ and coincides with that found by Perazzo and Gratton 13 in the Newtonian case.

Let $c_{1}=0$ in Eq. (4.4). In order to discuss the integrability of Eq. (4.4) we consider the following equation:

$$
\frac{\tan \alpha}{\epsilon} \int_{0}^{h} \frac{\xi^{2}}{\mu(\xi)} \mathrm{d} \xi-c h=0 .
$$

It is easy to check that Eq. (4.6), with $c<0$, does not admit a positive root so that Eq. (4.4) may be integrated numerically over the range $(0, \bar{h})$ for all $\bar{h}>0$. In this case the general solution is an increasing function defined over the interval $\left(c_{2},+\infty\right), c_{2}$ being an integration constant, tends to $+\infty$ as $s \rightarrow+\infty$, and its profile near the front $\left(s=c_{2}\right)$ is given by Eq. (4.5) 2 (see Figure 3).

On the contrary, Eq. (4.6), with $c>0$, may admit positive roots. If the pressure dependence of the viscosity upon pressure is of the form (2.14) with $n \in] 0,2]$, then Eq. (4.6) admits only one positive root that we call $h_{m}$ (see Figure 44). If viscosity depends on pressure according to (2.14) with $n>2$ or according to (2.13), then Eq. (4.6) admits two positive roots for some values of the nondimensional piezo-viscous coefficient $\lambda$ (we 


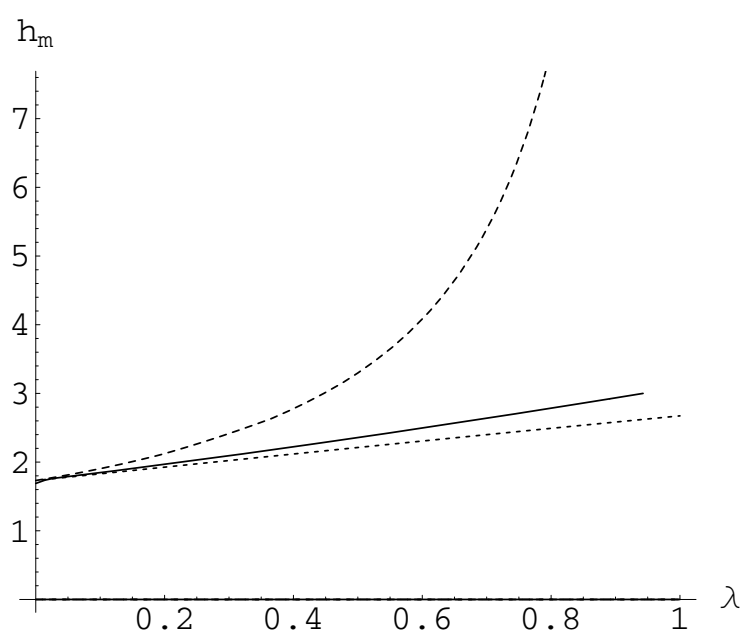

FIG. 4. Positive root $h_{m}$ of Eq. (4.6) as a function of the nondimensional piezo-viscous coefficient $\lambda$. Here we have assumed $\tan \alpha=\epsilon$, $c_{1}=0, c=1$ and $\mu(p)=1+\lambda p^{n}$, with $n=0.5$ (dotted line), $n=1$ (solid line), $n=2$ (dashed line). It is easy to check that $h_{m} \rightarrow(3 c \epsilon / \tan \alpha)^{-1 / 2}$, the unique positive root of Eq. (4.6) in the classical case $\mu(p)=1$, as $\lambda \rightarrow 0$. Furthermore, for $n=2, h_{m} \rightarrow+\infty$ as $\lambda \rightarrow \tan \alpha(\epsilon c)^{-1}$.

call $h_{m}$ the least positive root and $h_{M}$ the other), whereas it does not admit a positive root for other values of $\lambda$ (see Figure 5).

According to the number of positive roots of Eq. (4.6) one, two or three families of solutions to Eq. (4.4) may arise.

If Eq. (4.6) does not admit a positive root, then equation (4.4) may be numerically integrated over the range $(0, \bar{h})$ for all $\bar{h}>0$. In this case the general solution is a decreasing function defined over the interval $\left(-\infty, c_{2}\right), c_{2}$ being an integration constant, tends to $+\infty$ as $s \rightarrow-\infty$, and its profile near the front $\left(s=c_{2}\right)$ is given by Eq. (4.5) (see Figure 6).

If Eq. (4.6) admits only one positive root $h_{m}$, then two families of solutions to (4.4) arise. The first is formed by bounded decreasing functions defined over the range $\left(-\infty, c_{2}\right)$ satisfying the inequality $0 \leq h \leq h_{m}$. For these solutions we have $h \rightarrow h_{m}$ as $s \rightarrow-\infty$. Then they represent traveling waves behind a front running downslope, that far behind the front $(s \rightarrow-\infty)$ tend to the steady downslope flow $h=h_{m}$, and whose profile near the front $\left(s=c_{2}\right)$ is given by Eq. (4.5) 1 . The other family is formed by increasing functions bounded from below for which $h \geq h_{m}$. These solutions represents downslope traveling waves with no front for which $h \rightarrow h_{m}$ as $s \rightarrow-\infty$ and $h \rightarrow+\infty$ as $s \rightarrow+\infty$.

Finally, if Eq. (4.4) admits two positive solutions, $h_{m}<h_{M}$, as well as the downslope traveling waves behind a front, then the other two families of solutions to (4.4) representing downslope traveling waves with no front arise. The former is formed by bounded increasing functions satisfying the inequality $h_{m} \leq h \leq h_{M}$ and for which we have $h \rightarrow h_{m}$ as $s \rightarrow-\infty$ and $h \rightarrow h_{M}$ as $s \rightarrow+\infty$. The latter is formed by decreasing 


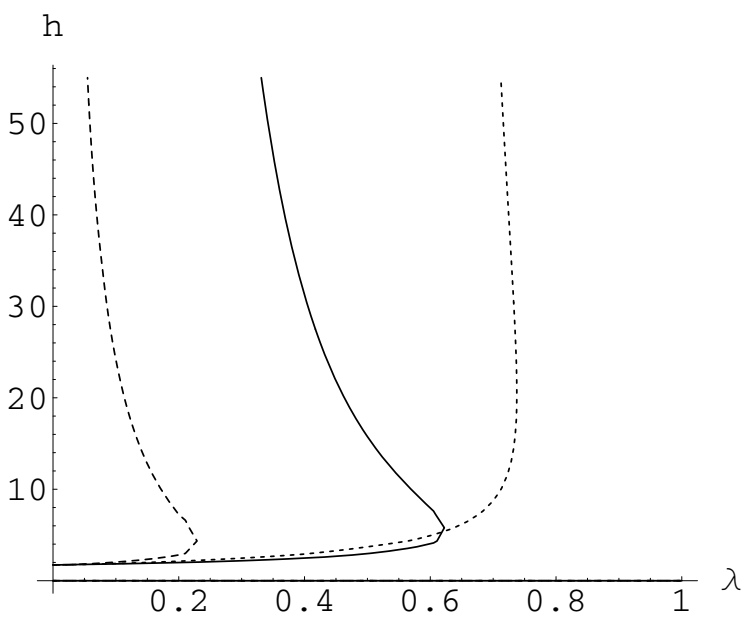

FIG. 5. Positive roots of Eq. 4.6, with $\tan \alpha=\epsilon, c_{1}=0$ and $c=1$. Here we have assumed the viscosity to depend on the pressure according to (2.14) with $n=2.1$ (dotted line), $n=3$ (dashed line), and according to (2.13) (solid line). In such a case $h_{M} \rightarrow+\infty$ as $\lambda \rightarrow 0^{+}$. On assuming an exponential dependence of the viscosity upon the pressure, in the case at hand, Eq. 4.6) does not admit a positive root for $\lambda>0.60216121$.

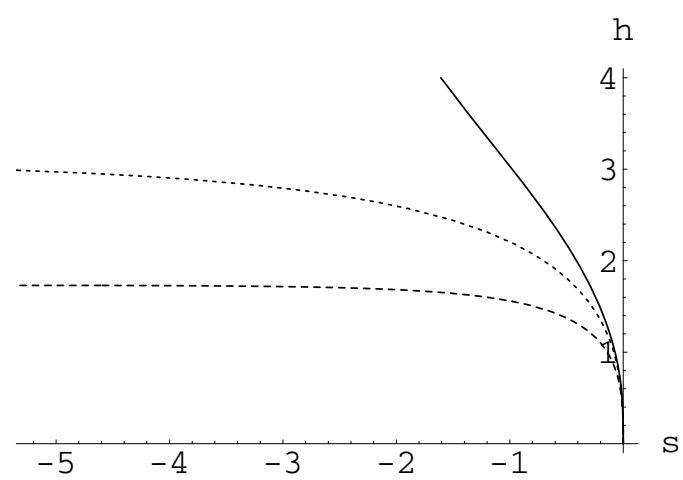

FIG. 6. Profiles of downslope traveling waves behind a front in the classical case $\mu(p)=1$ (dashed line), in the presence of a linear dependence of the viscosity on the pressure, $\mu(p)=1+p$ (dotted line), and in the presence of the nondimensional Barus' law $\mu(p)=$ $\mathrm{e}^{p}$. Here we have assumed $c=1, \tan \alpha=\epsilon$ and $c_{1}=c_{2}=0$.

functions that are bounded from below as they satisfy the inequality $h \geq h_{M}$ and for which we have $h \rightarrow+\infty$ as $s \rightarrow-\infty$ and $h \rightarrow h_{M}$ as $s \rightarrow+\infty$. 
4.2. Surface tension effect. If surface tension effects are included, then system (4.1) with the boundary conditions (2.8)-(2.10) and (2.16) admits the solution

$$
\left\{\begin{array}{l}
u=\left[\frac{\tan \alpha}{\epsilon}-\frac{\partial}{\partial x}\left(h-\frac{\Delta h}{\overline{\mathrm{Bo}}}\right)\right] \int_{0}^{z} \frac{h-\zeta}{\mu(h-\zeta-\Delta h / \overline{\mathrm{Bo}})} \mathrm{d} \zeta, \\
v=-\frac{\partial}{\partial y}\left(h-\frac{\Delta h}{\overline{\mathrm{Bo}}}\right) \int_{0}^{z} \frac{h-\zeta}{\mu(h-\zeta-\Delta h / \overline{\mathrm{Bo}})} \mathrm{d} \zeta, \\
p=h-z-\frac{\Delta h}{\overline{\mathrm{Bo}}}, \\
w=\int_{z}^{h} \nabla \cdot\left\{\int_{0}^{\zeta_{1}} \frac{h-\zeta_{2}}{\mu\left(h-\zeta_{2}-\Delta h / \overline{\mathrm{Bo}}\right)} \mathrm{d} \zeta_{2}\left[\frac{\tan \alpha}{\epsilon} \mathbf{i}-\nabla\left(h-\frac{\Delta h}{\overline{\mathrm{Bo}}}\right)\right]\right\} \mathrm{d} \zeta_{1} .
\end{array}\right.
$$

Then

$$
\begin{gathered}
h \bar{u}=\left[\frac{\tan \alpha}{\epsilon}-\frac{\partial}{\partial x}\left(h-\frac{\Delta h}{\overline{\mathrm{Bo}}}\right)\right] \int_{0}^{h} \frac{\xi^{2}}{\mu(\xi-\Delta h / \overline{\mathrm{Bo}})} \mathrm{d} \xi \\
h \bar{v}=-\frac{\partial}{\partial y}\left(h-\frac{\Delta h}{\overline{\mathrm{Bo}}}\right) \int_{0}^{h} \frac{\xi^{2}}{\mu(\xi-\Delta h / \overline{\mathrm{Bo}})} \mathrm{d} \xi
\end{gathered}
$$

and consequently the mass conservation equation (3.3) becomes

$$
h_{t}+\nabla \cdot\left\{\int_{0}^{h} \frac{\xi^{2}}{\mu(\xi-\Delta h / \overline{\mathrm{Bo}})} \mathrm{d} \xi\left[\frac{\tan \alpha}{\epsilon} \mathbf{i}-\nabla\left(h-\frac{\Delta h}{\overline{\mathrm{BO}}}\right)\right]\right\}=0 .
$$

It can be verified that in the classical case, viz. when the nondimensional viscosity $\mu(p)=1$, equation (4.7) differs from that studied by Brenner [5] only for the different nondimensionalization adopted.

If the surface tension effects are neglectable, equation (4.7) reduces to equation (4.2), that is, a nonlinear (forward) diffusion equation, and it is easy to show that no disturbance to the uniform solution $h=h_{0}$ grows in time. Surface tension acts through a fourth-order (forward) term so that no instabilities would occur. If equation (4.7) is linearized about $h=h_{0}$, one can seek periodic solutions of the resulting equation with periods $2 \pi / k_{x}$ and $2 \pi / k_{y}$ in the $x$ and $y$ directions $\left(k_{x}>0, k_{y}>0\right)$ of the form

$$
\tilde{h}=\exp \left[\mathrm{i}\left(k_{x} x+k_{y} y\right)+s t\right] .
$$

Denoting by $k=\sqrt{k_{x}^{2}+k_{y}^{2}}$ the two-dimensional wave number, the characteristic equation is given by

$$
\begin{aligned}
s= & -\mathrm{i} \frac{\tan \alpha}{\epsilon} k_{x}\left[\frac{h_{0}^{2}}{\mu\left(h_{0}\right)}+\frac{k^{2}}{\overline{\mathrm{Bo}}} \int_{0}^{h_{0}} \xi^{2} \frac{\mathrm{d}}{\mathrm{d} \xi}\left(\frac{1}{\mu}\right) \mathrm{d} \xi\right] \\
& -k^{2}\left(1+\frac{k^{2}}{\overline{\mathrm{Bo}}}\right) \int_{0}^{h_{0}} \frac{\xi^{2}}{\mu(\xi)} \mathrm{d} \xi,
\end{aligned}
$$

by which we deduce that the growth rate

$$
\operatorname{Re}(s)=-k^{2}\left(1+\frac{k^{2}}{\overline{\mathrm{Bo}}}\right) \int_{0}^{h_{0}} \frac{\xi^{2}}{\mu(\xi)} \mathrm{d} \xi
$$




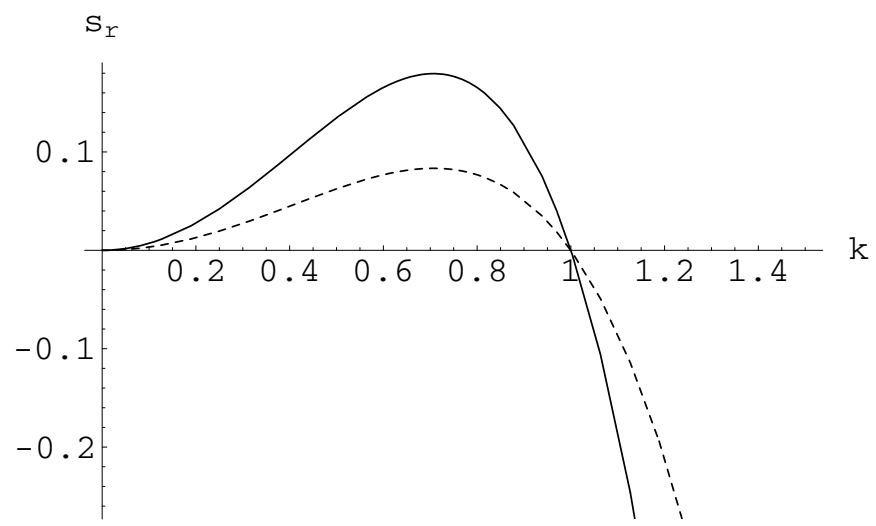

Fig. 7. Growth rate of the small perturbations to the uniform solution $h=1$ in the classical case $\mu(p)=1$ (dashed line) and in the presence of Barus' law $\mu(p)=\mathrm{e}^{p}$ (solid line). We have assumed that the normalized Bond number $\overline{\mathrm{Bo}}=1$. The positive value of $s_{\mathrm{r}}$ corresponds to the growth of a perturbation whereas the negative value of $s_{\mathrm{r}}$ indicates decay. Therefore in both cases the unstable domain is $0<k<\overline{\mathrm{Bo}}$.

is negative. Therefore, if at time $t=0$ a small bump is imposed to the free surface of the fluid, equation (4.10) governs how it will relax to $h=h_{0}$.

We now briefly examine the case in which the fluid moves on the underside of an incline. Here we replace $g$ with $-g$ in equation (2.1) 3 and find that the mass conservation equation is

$$
h_{t}+\nabla \cdot\left\{\int_{-h}^{0} \frac{\xi^{2}}{\mu(\xi-\Delta h / \overline{\mathrm{Bo}})} \mathrm{d} \xi\left[\frac{\tan \alpha}{\epsilon} \mathbf{i}+\nabla\left(h+\frac{\Delta h}{\overline{\mathrm{BO}}}\right)\right]\right\}=0 .
$$

If equation (4.11) is linearized about $h=h_{0}$, the growth rate of the periodic solutions (4.8) of the resulting equation satisfies the following relation:

$$
s_{\mathrm{r}}=\operatorname{Re}(s)=k^{2}\left(1-\frac{k^{2}}{\overline{\mathrm{Bo}}}\right) \int_{-h_{0}}^{0} \frac{\xi^{2}}{\mu(\xi)} \mathrm{d} \xi,
$$

by which we deduce that the layer is linearly unstable if

$$
k^{2}<k_{c}^{2}=\overline{\mathrm{Bo}},
$$

i.e., the perturbations are so long that the two-dimensional wave number is smaller than the normalized Bond number Bo (see Figure 7). In such a way we have generalized the result found by Oron et al. [12 for a fluid with constant viscosity to a fluid with a pressure-dependent viscosity.

5. Concluding remarks. A complete analysis of lubrication theory in the framework of piezo-viscous fluids has been considered. As a first step of our analysis a clear derivation of the governing equations and the boundary conditions has been provided. These equations may find interesting applications in geophysical shallow and slow flow, where the fluid depth is still important enough to allow significant variations of the 
pressure, but in any case the depth of the fluid is small with respect to the involved length.

To study these equations we have analyzed several flow regimes. In the quasi-steady regime, it is clear that the dependence of the viscosity on the pressure delays the breaking time of the flow pattern and therefore enhances the stability properties of the material flowing down the incline. This is an important finding which shows how the dependence of viscosity upon pressure is a fundamental point to understand the geophysical flows.

In the viscous regime, we have considered the propagation of traveling waves. In this case it is clear that the properties of the front of the wave are not influenced by the dependence of the viscosity on the pressure. This is because when $h \rightarrow 0$ the piezoviscosity law reduces to the classical one. The profiles of the traveling wave in the piezoviscous case may differ not only quantitatively but also qualitatively from the classical profiles. These phenomena also tell us that very compact geophysical flows may have unstable fronts, since in the front the piezo-viscous stabilizing effects disappear.

With respect to the stability of the uniform solution we find that the critical thresholds are the same as for the classical viscous theory, but in the interesting case of a flow in the bottom of the incline the amplitude of the perturbation grows in a much more consistent way when we consider a piezo-viscous fluid.

The next step is to investigate the effect of the dependence of constitutive parameters with respect to the pressure in a non-Newtonian framework, in such a way that a direct comparison of our findings with experimental data will be possible. This is because in geophysical applications the fluids involved have a complex rheology.

Acknowledgements. We thank Professor K.R. Rajagopal who has helped us to realize the importance of implicit constitutive equations in fluid mechanics and who provided us insight and expertise that greatly assisted our research. We thank an anonymous ref-

eree whose comments in a earlier version helped us to understand in a clearer way the important differences between the piezo-viscous theory and the classical lubrication approximation.

\section{REFERENCES}

[1] C. Ancey, Plasticity and geophysical flows: A review, J. Non-Newtonian Fluid Mech. 142 (2007), 4-35.

[2] E.C. Andrade, Viscosity of liquids, Nature, 125 (1930), 309-310.

[3] S. Bair, P. Kottke, Pressure-viscosity relationship for elastohydrodynamics, Tribology Trans., 46 (2003), 289-295.

[4] C. Barus, Isotherms, isopiestics and isometrics relative to viscosity, Amer. J. Sci. 45 (1893), 87-96.

[5] M.P. Brenner, Instability mechanism at driven contact lines, Phys. Rev. E, Vol. 47, No. 6 (1993), 4597-4599.

[6] J. Hron, J. Malek, K. R. Rajagopal: Simple flows of fluids with pressure-dependent viscosities. Proc. R. Soc. London A, 457 (2001) 1-20.

[7] H.M. Laun, Pressure dependent viscosity and dissipative heating in capillary rheometry of polymer melts, Rheol. Acta, 42, 295-308 (2003).

[8] J. Málek, J. Necas, K.R. Rajagopal: Global Analysis of the Flows of Fluids with Pressure-Dependent Viscosities, Arch. Rational Mech. Anal. 165 (2002), 243-269. MR1941479 (2004h:76003) 
[9] J. Málek, J. Necas, K.R. Rajagopal: Global Existence of Solutions for Flows of Fluids with Pressure and Shear Dependent Viscosities. Applied Mathematics Letters 15 (2002) 961-967. MR.1925921 (2003g:76032)

[10] M.J. Martín-Alfonso, F.J. Martínez-Boza, F.J. Navarro, M. Fernández, C. Gallegos, Pressuretemperature-viscosity relationship for heavy petroleum fractions, Fuel, 86 (2007), 227-233.

[11] J. Murali Krishnan, K.R. Rajagopal, Review of the uses and modeling of bitumen from ancient to modern times, Appl. Mech. Rev., 56 (2003), 149-214.

[12] A. Oron, S.H. Davis, S.G. Bankoff, Long-scale evolution of thin liquid films, Reviews of Modern Physics, Vol. 69, No. 3 (1997), 931-980.

[13] C.A. Perazzo, J. Gratton, Thin film of non-Newtonian fluid on an incline, Phys. Rev. E 67, 016307 (2003), 1-6.

[14] S.C. Prasad, K.R. Rajagopal, Flow of a fluid with pressure dependent viscosity due to a boundary that is being stretched, Applied Mathematics and Computation 173 (2006), 50-68. MR2203373 (2006h:76007)

[15] K.R. Rajagopal, On implicit constitutive theories for fluids, J. Fluid Mech. 550 (2006), $243-249$. MR2263984(2007e:76004)

[16] K.R. Rajagopal, A semi-inverse problem of flows of fluids with pressure-dependent viscosities, Inverse Probl. Sci. Eng. 16 No. 3 (2008), 269-280. MR2427495 (2009h:76062)

[17] K.R. Rajagopal, G. Saccomandi, Unsteady exact solution for flows of fluids with pressure-dependent viscosities, Mathematical Proceedings of the Royal Irish Academy 106A (2) (2006), 115-130. MR2266820 (2008c:35255)

[18] K.R. Rajagopal, G. Saccomandi, L. Vergori,On the Oberbeck-Boussinesq approximation in fluids with pressure-dependent viscosities, Nonlinear Analysis: Real World Applications 10 (2009), No. 2 1139-1150. MR2474287

[19] K.R. Rajagopal, G. Saccomandi, L. Vergori, Stability analysis of the Rayleigh-Bénard convection for a fluid with temperature and pressure dependent viscosity, Z. Angew. Math. Phys. 60 (2009), 739-755. MR2520609 (2010g:76058)

[20] K.R. Rajagopal, A.Z. Szeri, On an inconsistency in the derivation of the equations of elastohydrodynamic lubrication, Proc. R. Soc. A 459 (2003), 2771-2786. MR2015989(2004i:76069)

[21] R.N.J. Saal, G. Koens, Investigations into plastic properties of asphaltic bitumen, Physics, 7 (1933), 408-412.

[22] R.N.J. Saal, J.W.A. Labout, Rheological properties of asphalt, in: F.R. Eirich (Ed.) Rheology: Theory and Applications, Academic Press, New York, 363-400, 1958.

[23] G.G. Stokes, On the theories of the internal friction of fluids in motion, and motion of elastic solids, Trans. Camb. Phil. Soc. 8 (1845), 287-305.

[24] S.C. Subramanian, K.R. Rajagopal, A note on the flow through porous solids at high pressures, Comput. Math. Appl. 53 (2007), 260-275. doi:10.1016/j.camwa.2006.02.023. MR2325232

[25] A.Z. Szeri, Fluid Film Lubrication: Theory and Design, Cambridge University Press, 1998.

[26] M. Vasudevaiah, K.R. Rajagopal, On fully developed flows of fluids with a pressure dependent viscosity in a pipe, Applications of Mathematics 50, No. 4 (2005), 341-353. MR2151461|(2006b:76002) 